\title{
Suicide preventing actions in schizophrenia
}

\author{
N Dissaux ${ }^{1}$, S Berrouiguet ${ }^{1}$, M Walter $^{1}$, C Lemey $^{1}$ \\ ${ }^{1}$ Brest Medical University Hospital, Psychiatry Department \\ mail : nolwenn.dissaux@chu-brest.fr
}

\section{INTRODUCTION}

During the 20th century, improvements in schizophrenia treatments have allowed to reduce long-term disability and to improve patient's quality of life.

However, suicide rate in people with schizophrenia remains significantly higher than in general population.

Lifetime suicide risk is estimated around $10 \%$ in this population. It is also known that $\mathbf{5 0 \%}$ of patients with schizophrenia attempt suicide in their lifetime.

Many studies have been conducted to identify suicide risk factors in schizophrenia. The aim is to develop strategies to reduce the incidence of suicide behaviors in this population.

Our purpose is to determine whether some specific strategies have proven evidence of efficacy for suicide reduction in schizophrenia.

\section{METHOD}

A literature search of articles on PubMed and PsycINFO, published in English up to February 2019, was performed using the following terms " schizophren* AND suicid* AND (prevent* OR intervent* OR strateg* OR therap*).

Articles were selected if they provided a full description and/or an assessment of a specific strategy for suicide prevention in schizophrenia.

\section{RESULTS}

Out of 27, 6 articles were selected :

- 4 of them assess a psychotropic drug

- 1 evaluates the effect of a specific follow-up at the beginning of the illness

- 1 evaluates the role of a cognitive behavioral therapy (CBT) for patients with schizophrenia resistant to conventional antipsychotic medication

\section{Drugs:}

Clozapine is the only drug that gathered evidence as an effective treatment for reducing suicide risk in schizophrenia.

A randomized controlled study of Clozapine vs Olanzapine shows that Clozapine efficacy for suicide prevention is not related to the increased clinical contact due to white blood cell count monitoring. However, the pharmacological mechanism for the effect of Clozapine is not yet understood.
Figure 1. Flow chart

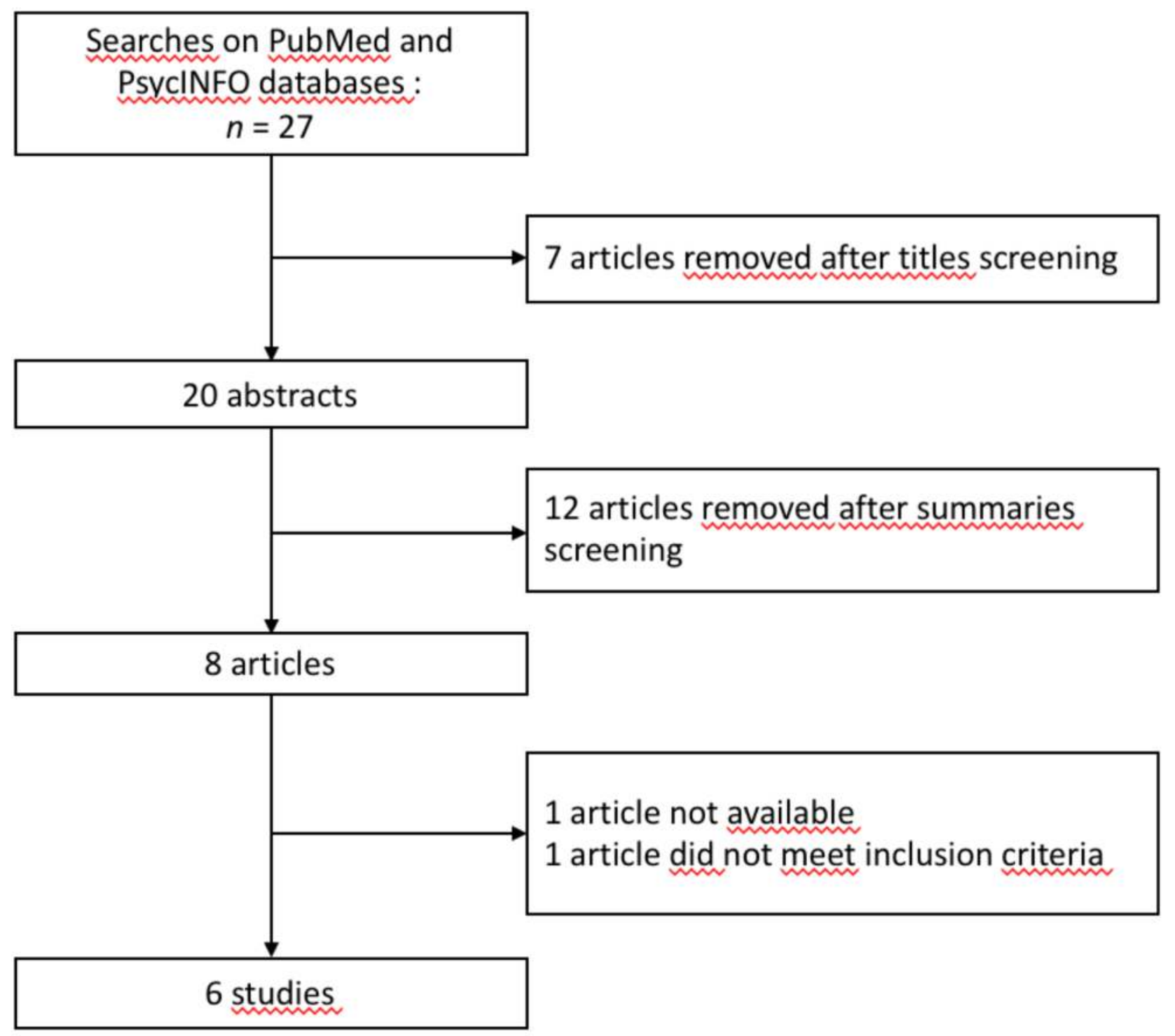

Specific follow-up:

A 2-year early intervention program in patients with first episode schizophrenia spectrum disorders found that patients who underwent the program had significantly fewer suicides than patients with a classical follow-up. This effect lasted a few years after the end of the program.

\section{Cognitive Behavioral Therapies:}

A randomized controlled study demonstrates that CBT provides significant reductions of suicidal ideations in comparison to befriending.

\section{DISCUSSION AND CONCLUSION}

Most programs proposed these last years concentrate on first episode psychosis, our aim was to explore preventing actions during the development of the disease.

Surprisingly, considering the seriousness of the outcome, this literature search shows that very few studies have been conducted to evaluate suicide preventing actions in schizophrenia.

Evidence of efficacy for suicide prevention is difficult to obtain, even in general population, as suicide is a rare event.

Clozapine appears to be the elected treatment for patients with schizophrenia who present many risk factors of suicide.

However, studies evaluating suicide risk factors in schizophrenia emphasize that hopelessness is a key determinant of suicide.

Preventing actions should be develop to target this hopelessness feeling. 